\title{
DEVELOPMENT OF A MATHEMATICAL MODEL OF THE FUNCTIONING OF MULTI-ANTENNA RADIO COMMUNICATIONS WITH SPECTRALLY EFFECTIVE SIGNALS UNDER THE INFLUENCE OF DESTABILIZING FACTORS
}

\author{
Viktor Ostapchuk ${ }^{1}$, Oleg Sova ${ }^{2}$, Andrii Shyshatskyi ${ }^{3}$, Olexander Harashchuk ${ }^{4}$ \\ Military institute of telecommunications and informatization named after Heroes of Kruty, Kyiv, Ukraine \\ vikost021@gmail.com \\ ORCID: http://orcid.org/0000-0001-5686-0198 \\ ${ }^{2}$ Department of automated control systems, Military institute of telecommunications and informatization named after Heroes of Kruty, \\ Kyiv, Ukraine \\ soy_135@ukr.net \\ ORCID: http://orcid.org/0000-0002-7200-8955 \\ ${ }^{3}$ Research department of electronic warfare development, Central scientifically-research institute of arming and military equipment of \\ the Armed Forces of Ukraine, Kyiv, Ukraine \\ ierikon13@gmail.com \\ ORCID: http://orcid.org/0000-0001-6731-6390 \\ ${ }^{4}$ National Defense University of Ukraine named after Ivan Cherniakhovskyi, Kyiv, Ukraine \\ svasist@ukr.net \\ ORCID: http://orcid.org/0000-0003-3104-8341
}

ARTICLE INFO

Article history:

Received date 07.07.2020

Accepted date 30.07.2020

Published date 31.08 .2020

Section:

Information Technology

DOI

$10.21303 / 2313-8416.2020 .001393$

\section{KEYWORDS}

programmable radio equipment spectrally effective signals

efficiency

multi-antenna systems

\section{ABSTRACT}

The necessity of developing a mathematical model for the functioning of multi-antenna radio communication facilities with spectrally effective signals under the influence of destabilizing factors has been substantiated. The results of the study of the influence of destabilizing factors on the multi-antenna systems with spectrally effective signals are presented. New analytical dependences have been obtained, which make it possible to calculate the effect of destabilizing factors on the efficiency of multi-antenna system with spectrally effective signals.

Object of research: development of a mathematical model of the functioning of multi-antenna radio communication facilities with spectrally effective signals under the influence of destabilizing factors.

Investigated problem: taking into account additional destabilizing factors on the multi-antenna of a radio communication facility with spectrally effective signals.

Main scientific results: when transmitting information in multi-antenna radio communication systems with spectrally effective signals, the following are taken into account: Type of parameters of deliberate interference, type and parameters of deliberate fading, type and parameters of security code structures, phase jitter, slope of the constellation matrix, Intersymbol interference, high mobility of objects (transmitter and receiver), the number of receiving and transmitting antennas, the number of subcarriers in the antenna channel. It has been established that taking into account all the parameters in the system makes it possible to assess the state of the channel, to develop measures to reduce the negative influence of destabilizing factors on the noise immunity of multi-antenna systems.

A review of the practical use of research results: radio communication facilities with programmable architecture.

Innovative technologies product: technology for substantiating the architecture of transceivers, software for programmable radio equipment, which can increase the noise immunity of radio communications, taking into account additional destabilizing factors and software for radio monitoring equipment.

Overview of an innovative technological product: programmable radios, software for radio communications.

(C) The Author(s) 2020. This is an open access article under the CC BY license http://creativecommons.org/licenses/by/4.0).

\section{Introduction}

MIMO (Multiple Input Multiple Output) technology has found practical application in many modern telecommunication systems. MIMO technology is used in wireless local area 
networks of the IEEE 802.11x standard, as well as in wireless mobile networks WIMAX and LTE, etc. [1, 2].

Analysis of recent local conflicts has shown that they most often occur in cities. This leads to an increase in the requirements for the stability of radio communications, which is associated with the high density of buildings and high mobility of subscribers.

As an alternative to the Orthogonal Frequency Division Multiplexing (OFDM) technology $[3,4]$, in order to increase the frequency efficiency of the channels, it is proposed to use Spectrally Efficient Frequency Division Multiplexing (SEFDM) signals [5, 6].

An increase in the spectral efficiency of SEFDM signal designs is achieved by moving to non-orthogonal frequency division multiplexing of subcarriers.

However, the analysis of SEFDM-signaling structures revealed the following disadvantages [3, 4]: high level of intersymbol interference in the channel; exactingness to the synchronization system; high peak-factor; high computational complexity (depending on the number of subcarriers and on the volume of the channel alphabet of subcarriers) and low noise immunity.

The above stipulates the search for new scientific approaches to increase the energy and frequency efficiency of multi-antenna systems using hybrid information technology MIMO-SEFDM.

All this confirms the relevance of the chosen research direction.

\section{1. The object of research}

The object of research is the MIMO-SEFDM radio communication systems operating in conditions of interference and signal fading.

\section{2. Problem description}

In work [4] the development of a geometric model of the state of the system channel by. The proposed model was developed to take into account the movement of vehicles when transferring information through the channels of the system. However, the proposed model is based only on one ring of scatterers and does not take into account the influence of deliberate interference and other destabilizing factors.

The work [7] is devoted to the development of a complex model for describing the state of the channel of multi-antenna systems. However, the specified model does not take into account the influence of intentional interference, and the mathematical model is also indicated does not work at a low signal-to-noise ratio.

In the known publications, there is no analysis of the noise immunity of SEFDM signal structures, however, in the articles [5, 6], separate curves of the potential noise immunity of receiving SEFDM signals without noise-immune coding with coherent reception, optimal according to the criterion of the minimum average error probability per SEFDM-symbol, are given.

Also in $[6,7]$, the assessment of potential noise immunity was carried out by one or two values of the number of subcarriers and only at two values of their frequency spacing, without the influence of deliberate interference.

In [8] and [9], the comparison of the noise immunity of receiving SEFDM signals at was carried out with the potential noise immunity of single-frequency signals, which is incorrect.

In this case, in all the considered sources, the noise immunity of reception was considered for low-position phase-shift keyed (PK) signals under the influence of additive white Gaussian noise (AWGN).

\section{3. Proposed solution to the problem}

In order to increase the energy and frequency efficiency of radio communication systems, it is proposed to jointly use multi-antenna systems with spectrally efficient signals.

That is why the aim of research is to develop a mathematical model of the functioning of multi-antenna radio communication facilities with spectrally effective signals under the influence of destabilizing factors.

\section{Materials and methods}

For a scientific task, the authors used the main provisions of the theory of noise immunity, electronic protection and multidimensional optimization. 


\section{Research results}

The model of a multipath channel with spectrally efficient signals is presented as a model of a channel with discrete multi-beam according to $[5,9]$.

$$
z_{t}(t)=\operatorname{Re}\left[\sum_{H_{\min }}^{H_{\max }}\left(C_{l, \partial, r} \times \psi_{r, c, l}\right) \sum_{\xi=1 n=1}^{\equiv} \sum_{n=1}^{N_{t}}\left[\begin{array}{l}
k_{c \ln } u_{x}\left(t-(s-1) T-\tau_{l}\right)+ \\
+k_{s \ln } u_{x}\left(t-(s-1) T-\tau_{l}\right)+n(t)
\end{array}\right]\right], 0<t<\infty,
$$

where $k_{s \ln }, k_{c \mid n}$ - the random in-phase and quadrature components of the transmission coefficient in the $l$-th beams from the $s$-th transmitting element, $\Xi$ - the total number of beams in the channel, $\tau_{l}$ - the delay time in the $l$-th beams, $u_{x}$ - the known realizations of signals at the receiver input, $x=(0,1, \ldots, M-1)-$ sequence of information symbols, M-positionality of signal manipulation, $T-\mathrm{du}-$ ration of the transmission of signal elements, $n(t)$ - additive white Gaussian noise, $C_{l, \partial, r}$ - value of the point of the signal-code structure for the $l$-th beam in the frame $r$ symbol $\partial$;

$$
\begin{gathered}
\emptyset_{r, \partial, l}(t)=\left\{\begin{array}{l}
\left(\left(t-s T_{\partial}-\Xi T_{\partial}\right) / T_{u}\right) \text { for }(s+N r) T_{s} \leq t \leq\left(\Xi_{r}+1\right) T_{\partial}, \\
0 \text { otherwise, }
\end{array}\right. \\
l^{\prime}=l-\left(H_{\max }+H_{\min }\right) / 2, \\
T_{\partial}=T_{u}+T_{g},
\end{gathered}
$$

where $H_{\min }$ and $H_{\max }$ - the minimum and maximum value of the MIMO-SEFDM channel (lower and upper bounds); $r$ - number of the MIMO-SEFDM transmission frame, $T_{u}$ - duration of the useful part of the MIMO-SEFDM frame, $T_{\partial}$ - duration of the MIMO-SEFDM frame, $f$ - reference frequency of the transmitter of the MIMO-SEFDM system.

Let's consider the process of passing one MIMO-SEFDM element $\left(t \in\left[0 ; N T_{\partial}\right]\right)[8,10]$.

The signal $z^{*}(t)$ at the receiver input has the form

$$
z^{*}(t)=z(t)+n(t)
$$

where $n(t)$ - the function describing the noise in the channel, consisting of a useful signal $z(t)$, which can be represented as a ratio:

$$
C_{\partial, l}^{*}=C_{\partial, l}+n_{\partial, l},
$$

where $n_{\partial, l}$ - the component $n(t)$ superimposed on $C_{\partial, 1}$ in the MIMO-SEFDM channel $\partial$ symbol $\partial$ after transformation.

Since $C_{\partial, l}^{*}$ is a complex number, its real and imaginary parts in (4) are conveniently represented as a matrix of channel components:

$$
\left(\begin{array}{l}
\operatorname{Re}\left\{C_{\partial, l}^{*}\right\} \\
\operatorname{Im}\left\{C_{\partial, l}^{*}\right\}
\end{array}\right)=\left(\begin{array}{l}
\operatorname{Re}\left\{C_{\partial, l}\right\} \\
\operatorname{Im}\left\{C_{\partial, l}\right\}
\end{array}\right)+\left(\begin{array}{l}
\operatorname{Re}\left\{n_{\partial, l}\right\} \\
\operatorname{Im}\left\{n_{\partial, l}\right\}
\end{array}\right) .
$$

Since the linear transformation matrix is invertible, its geometric distortions are similar to the sequence of reflections, rotations, expansions and shifts. In our case, reflections are impossible, since the interference is not capable of causing such a transformation. The other three transformations correspond to phase shift, amplitude mismatch, and quadrature error. There is also intersymbol interference, phase jitter and Gaussian noise in a typical channel.

Let's consider each of the transformations

$$
\left(\operatorname{Re}\left\{C_{\partial, l}\right\} \operatorname{Im}\left\{C_{\partial, l}\right\}\right)^{\mathrm{T}} \rightarrow\left(\operatorname{Re}\left\{C_{\partial, l}^{*}\right\} \operatorname{Im}\left\{C_{\partial, l}^{*}\right\}\right)^{\mathrm{T}}
$$

in the absence of $n_{\partial, l}$. 
The phase shift is a deterministic phase error, which is the rotation of the constellation diagram around an axis by an angle $\theta_{\text {const }}$ :

$$
\left(\begin{array}{l}
\operatorname{Re}\left\{C_{\partial, l}^{*}\right\} \\
\operatorname{Im}\left\{C_{\partial, l}^{*}\right\}
\end{array}\right)=\left(\begin{array}{cc}
\cos \theta_{\text {const }} & -\sin \theta_{\text {const }} \\
\sin \theta_{\text {const }} & \cos \theta_{\text {const }}
\end{array}\right)\left(\begin{array}{l}
\operatorname{Re}\left\{C_{\partial, l}\right\} \\
\operatorname{Im}\left\{C_{\partial, l}\right\}
\end{array}\right) .
$$

The amplitude mismatch is realized by introducing a gain coefficient $k_{E}$ for the actual channel that is different from the corresponding gain of the imaginary channel, that is

$$
\left(\begin{array}{l}
\operatorname{Re}\left\{C_{\partial, l}^{*}\right\} \\
\operatorname{Im}\left\{C_{\partial, l}^{*}\right\}
\end{array}\right)=\left(\begin{array}{l}
\operatorname{Re}\left\{C_{\partial, l}\right\} \\
\operatorname{Im}\left\{C_{\partial, l}\right\}
\end{array}\right)+\left(\begin{array}{l}
\operatorname{Re}\left\{n_{\partial, l}\right\} \\
\operatorname{Im}\left\{n_{\partial, l}\right\}
\end{array}\right)
$$

Quadrature error is the result of matrix multiplication, causing the slope of the constellation diagram relative to the reference value

$$
\left(\begin{array}{l}
\operatorname{Re}\left\{C_{\partial, l}^{*}\right\} \\
\operatorname{Im}\left\{C_{\partial, l}^{*}\right\}
\end{array}\right)=\left(\begin{array}{cc}
\cos \theta_{\text {const }} & -\sin \theta_{\text {const }} \\
\sin \theta_{\text {const }} & \cos \theta_{\text {const }}
\end{array}\right)\left(\begin{array}{l}
\operatorname{Re}\left\{C_{\partial, l}\right\} \\
\operatorname{Im}\left\{C_{\partial, l}\right\}
\end{array}\right) .
$$
channel.

$k_{s}$ - inclination angle from the orthogonality of the imaginary and real components of the

Let's divide the component $n_{\partial, l}$ into two components, one of which $\left(n_{\partial}\right)$ is associated with intersymbol interference in the symbol $\partial_{s}$, the other $\left(n_{l}\right)$ is additive white Gaussian noise in the MIMO-SEFDM channel. Considering that the interference is caused by a false signal, which causes a shift of symbols in the constellation diagram, it is modeled by a false signal vector with amplitude $\mathrm{A}$ and phase $\varphi$, which depends on the moment of measurement and the frequency difference of the false and useful signals.

Then the matrix will look like:

$$
\left(\begin{array}{l}
\operatorname{Re}\left\{n_{\partial, l}\right\} \\
\operatorname{Im}\left\{n_{\partial, l}\right\}
\end{array}\right)=\left(\begin{array}{c}
A \cos \phi \\
A \sin \phi
\end{array}\right)+\left(\begin{array}{c}
\operatorname{Re}\left\{n_{l}\right\} \\
\operatorname{Im}\left\{n_{l}\right\}
\end{array}\right) .
$$

Phase jitter, in contrast to phase shift, amplitude mismatch and quadrature error, is a random error and causes the constellation diagram to rotate through an angle $\theta_{i}$ that is a random variable with a Gaussian distribution and zero mean and variance $\sigma_{i}^{2}$, that is.

So

$$
\left(\begin{array}{l}
\operatorname{Re}\left\{C_{\partial, l}^{*}\right\} \\
\operatorname{Im}\left\{C_{\partial, l}^{*}\right\}
\end{array}\right)=\left(\begin{array}{cc}
\cos \theta_{i} & -\sin \theta_{i} \\
\sin \theta_{i} & \cos \theta_{i}
\end{array}\right)\left(\begin{array}{l}
\operatorname{Re}\left\{C_{\partial, l}\right\} \\
\operatorname{Im}\left\{C_{\partial, l}\right\}
\end{array}\right)
$$

Generalizing (6), taking into account (9)-(13), let's obtain:

$$
\begin{aligned}
& \left(\begin{array}{l}
\operatorname{Re}\left\{C_{\partial, l}^{*}\right\} \\
\operatorname{Im}\left\{C_{\partial, l}^{*}\right\}
\end{array}\right)=K\left(\begin{array}{cc}
\cos \theta_{i} & -\sin \theta_{i} \\
\sin \theta_{i} & \cos \theta_{i}
\end{array}\right)\left(\begin{array}{cc}
\cos \theta_{\text {const }} & -\sin \theta_{\text {const }} \\
\sin \theta_{\text {const }} & \cos \theta_{\text {const }}
\end{array}\right) \times \\
& \times\left(\begin{array}{cc}
k_{E} & 0 \\
0 & 1
\end{array}\right)\left(\begin{array}{cc}
1 & k_{s} \\
0 & 1
\end{array}\right)\left(\begin{array}{l}
\operatorname{Re}\left\{C_{\partial, l}\right\} \\
\operatorname{Im}\left\{C_{\partial, l}\right\}
\end{array}\right)+\left(\begin{array}{l}
A \cos \phi \\
A \sin \phi
\end{array}\right)+\left(\begin{array}{l}
\operatorname{Re}\left\{n_{l}\right\} \\
\operatorname{Im}\left\{n_{l}\right\}
\end{array}\right) .
\end{aligned}
$$

Approximating relation (12) for random phase angles and the influence of deliberate interference, let's obtain the following mathematical expectation of the statistical moments of the received symbols $C_{\partial, l}^{*}$

$$
\begin{gathered}
M\left[\operatorname{Re}\left(C_{\partial, l}^{*}\right)=M\left[K k_{E} \operatorname{Re}\left\{C_{\partial, l}\right\}-K\left(k_{E} k_{s}-\theta_{\text {const }}\right) \operatorname{Im}\left\{C_{\partial, l}\right\}\right]\right], \\
M\left[\operatorname{Im}\left(C_{\partial, l}^{*}\right)=M\left[K k_{E} \theta_{\text {const }} \operatorname{Re}\left\{C_{\partial, l}\right\}-K\left(k_{E} k_{s}-\theta_{\text {const }}+1\right) \operatorname{Im}\left\{C_{\partial, l}\right\}\right]\right] .
\end{gathered}
$$


Phase jitter is determined by sequentially calculating the covariance of the two components of the received symbol $C_{\partial, l}^{*}$ :

$$
\operatorname{Cov}\left[\operatorname{Re}\left\{C_{\partial, l}^{*}\right\}, \operatorname{Im}\left\{C_{\partial, l}^{*}\right\}\right]=-K^{2} k_{E} \sigma_{i}^{2}\left(\operatorname{Re}\left\{C_{\partial, l}\right\} \operatorname{Im}\left\{C_{\partial, l}\right\}+\left[\operatorname{Re}\left\{C_{\partial, l}\right\}\right]^{2} k_{s}\right)
$$

\section{Discussion of the results on the development of a mathematical model}

A complex mathematical model for assessing the state of the channel of multi-antenna radio communication systems is proposed. The main advantages of the proposed model are:

- unambiguity of the obtained estimate of the channel state;

- wide scope of use (radio communication and radar systems);

- ability to adapt to the signaling environment in the channel;

- greater accuracy of channel state estimation;

- possibility of synthesizing the optimal structure of a radio communication facility.

The advantages of this model are due to the fact that a greater number of destabilizing factors are taken into account than the known ones. The model takes into account in the complex intentional interference of an additive and multiplicative nature, destabilizing factors due to the mutual movement of transmitters and receivers. The influence of deliberate interference, the slope of the channel constellation matrix, intersymbol interference, phase jitter, the type of security code structure and the effect of signal fading.

The disadvantages of the proposed complex mathematical model should be considered a great computational complexity compared to simpler mathematical models. This is due to the calculation of a larger number of channel state indicators.

The specified mathematical model is advisable to use in radio stations with programmable architecture, it functions in conditions of active electronic suppression.

The specified complex mathematical model will allow:

- identify the structure of the interference, its type and the law of setting;

- assess the state of the channel;

- use effective signal-code structures to ensure channel noise immunity;

- ensure efficient use of the radio frequency resource of programmable radio communications;

- increase the speed of evaluation of communication channels;

- develop measures aimed at increasing the noise immunity.

The complex mathematical model proposed in the work is expedient to use in the development of software for modules (blocks) for evaluating promising radio communications, based on the interfaces of the open architecture of the SCA 2.2 version.

Directions for further research should be aimed at reducing computational costs and taking into account the influence of non-Gaussian interference.

\section{Conclusions}

1 The article deals with the development of a mathematical model of the functioning of multi-antenna radio communication facilities with spectrally effective signals under the influence of destabilizing factors.

The difference between the proposed mathematical model lies in the establishment of new analytical relations, additionally take into account the type and parameters of deliberate interference, the type and parameters of deliberate fading, the type and parameters of security code structures, phase jitter, slope of the constellation matrix, Intersymbol interference, high mobility of objects (transmitter and receiver), the number of receiving and transmitting antennas, the number of subcarriers in the antenna channel.

2. The proposed mathematical model of the channel state of multi-antenna radio communication systems with spectrally effective signals is more accurate than the known ones, due to taking into account a larger number of destabilizing factors, which in turn leads to an increase in computational complexity.

3. The proposed model is advisable to use when developing software for modern radio stations and when developing software for radio monitoring tools. 


\section{References}

[1] Sliusar, V. (2005). Cistemy MIMO: printsipy postroeniia i obrabotka signalov. Elektronika: Nauka, Tekhnologiia, Biznes, 8, 52-58.

[2] Kuvshynov, O. V. (2009). Adaptyvne upravlinnia zasobamy zavadozakhystu viiskovykh system radiozviazku. Zbirnyk naukovykh prats VIKNU, 17, 125-130.

[3] Jia, R., Li, Y., Cheng, X., Ai, B. (2018). 3D geometry-based UAV-MIMO channel modeling and simulation. China Communications, 15 (12), 64-74.

[4] Ma, Y., Yang, L., Zheng, X. (2018). A geometry-based non-stationary MIMO channel model for vehicular communications. China Communications, 15 (7), 30-38. doi: http://doi.org/10.1109/cc.2018.8424580

[5] Zhu, Q., Jiang, K., Chen, X., Zhong, W., Yang, Y. (2018). A novel 3D non-stationary UAV-MIMO channel model and its statistical properties. China Communications, 15 (12), 147-158.

[6] Shyshatskyi, A. V., Bashkyrov, O. M., Kostyna, O. M. (2015). Rozvytok intehrovanykh system zviazku ta peredachi danykh dlia potreb Zbroinykh Syl. Ozbroiennia ta viiskova tekhnika, 1 (5), 35-40.

[7] Zhyvotovskyi, R. M., Shyshatskyi, A. V., Petruk, S. N. (2017). Structural-semantic model of communication channel. Problems of Infocommunications. Science and Technology. Kharkiv, 524-529. doi: http://doi.org/10.1109/infocommst.2017.8246454

[8] Goldsmith, A., Jafar, S. A., Jindal, N., Vishwanath, S. (2003). Capacity limits of MIMO channels. IEEE Journal on Selected Areas in Communications, 21 (5), 684-702. doi: http://doi.org/10.1109/jsac.2003.810294

[9] Kalantaievska, S., Pievtsov, H., Kuvshynov, O., Shyshatskyi, A., Yarosh, S., Gatsenko, S. et. al. (2018). Method of integral estimation of channel state in the multiantenna radio communication systems. Eastern-European Journal of Enterprise Technologies, 5 (9 (95)), 60-76. doi: http://doi.org/10.15587/1729-4061.2018.144085

[10] Petruk, S. N., Zhyvotovskyi, R. M., Shyshatskyi, A. V. (2018). Mathematical Model of MIMO. Problems of Infocommunications. Science and Technology. Kharkiv, 7-12. doi: http://doi.org/10.1109/infocommst.2018.8632163 